\title{
Indefinite Metrik im Zustandsraum und Wahrscheinlichkeitsinterpretation
}

\author{
Von Siegrried Schlieder \\ Aus dem Max-Planck-Institut für Physik und Astrophysik, München \\ (Z. Naturforschg. 15 a, 555-565 [1960] ; eingegangen am 3. März 1960)
}

\begin{abstract}
The content of the part I was the construction of the fundamental metric tensor; in the part II the conditions which make possible the probability interpretation for elements lying in the same coherent sector were discussed. The subject of the following part is the space of states as a whole. The existence of superselection rules allows one to give up the fundamental tensors formerly introduced as identity matrix in the subspace of the physical states. The mathematical form, in which symmetry groups appear is shown by an example. Finally a possible generalization for the dual element of an element is given.
\end{abstract}

\section{Teil III: Übergang vom kohärenten Sektor zum ganzen Zustandsraum}

\section{$\S 1$. Überauswahlregel}

Nunmehr sollen die Transformationsgruppen besprochen werden, deren Transformationen Abbildungen eines kohärenten Sektors auf einen anderen bewirken oder innerhalb eines kohärenten Sektors triviale Abbildungen liefern. Ehe Beispiele für solche Symmetriegruppen folgen, soll zuerst der Zerfall des Zustandsraumes in kohärente Sektoren, infolge von Überauswahlregeln erläutert werden.

Überauswahlregeln treten zusammen mit gewissen Operatoren $B_{k}$ auf, die durch eine bemerkenswerte Eigenschaft ausgezeichnet sind: Die $B_{k}$ sollen mit allen Operatoren $A_{m}$, welche meßbaren Größen zugeordnet sind, vertauschen.

$$
\left[B_{k}, A_{m}\right]_{-}=0 .
$$

Die $B_{k}$ haben stets Auswahlregeln bzw. einen Erhaltungssatz zur Folge, denn $B_{k}$ vertauscht insbesondere mit $H$, dem Hamilton-Operator $\left[B_{k}, H\right]_{-}=0$. Das umgekehrte ist natürlich nicht der Fall: So gilt z. B. für die Impulskomponenten $p_{k}$ wegen $\left[p_{k}, H\right]_{-}=0$ der bekannte Erhaltungssatz, aber für die Impulskomponenten gilt keine UUberauswahlregel, da eine Ortsmessung den Zustand in eine Superposition von Zuständen mit verschiedenen Impulseigenwerten überführt.

Die geforderte Vertauschungseigenschaft der $B_{k}$ mit den Meßoperatoren bewirkt, daß ein Zustand $|\varphi\rangle$ und ein Zustand $B_{k}|\varphi\rangle$ durch Messungen nicht zu unterscheiden, d. h. physikalisch identisch sind.

Wenn man außerdem fordert, daß ein physikalischer Zustand durch Messungen bis auf eine komplexe Konstante und bei Normierung bis auf eine komplexe Konstante vom Betrag 1 festgelegt ist, folgt daraus, daß im Falle der Beibehaltung der Normierung $B_{k}|\varphi\rangle=e^{i \lambda}|\varphi\rangle$ ( $\lambda$ reell) sein muß. Die tatsächlich vorkommenden Operatoren $B_{k}$ haben auch diese Eigenschaft, z. B. der Operator $T^{2}$, wobei $T$ der Zeitumkehrtransformation

$$
\left(\begin{array}{cccc}
1 & & & 0 \\
& 1 & 1 & \\
0 & & & -1
\end{array}\right)
$$

zugeordnet ist. $T^{2}$ besitzt für halbzahlige Spinzustände den Eigenwert -1 , für ganzzahlige den Eigenwert +1 .
Dieselbe Eigenschaft haben auch die Operatoren $\Pi^{2}$, die zu Drehungen um einen Winkel $2 \pi$ gehören. Des weiteren fallen hierunter spezielle Eichtransformationen im Zustandsraum, z. B. die Transformation $|\varphi\rangle \rightarrow e^{i \alpha q}|\varphi\rangle$, wobei $q$ der Operator der elektrischen Ladung, $\alpha$ eine reelle Zahl ist. Für die Baryonenzahl $N$ existiert eine entsprechende Transformation

$$
|\varphi\rangle \rightarrow e^{i \alpha N}|\varphi\rangle,
$$

wahrscheinlich auch für die Leptonenzahl $\mathbb{\Omega}$

$$
|\varphi\rangle \rightarrow e^{i \alpha \mathfrak{L}}|\varphi\rangle \text {. }
$$

Das Postulat für die hingeschriebenen Operatoren der Eichtransformationen ist ja gerade, daß ihre Anwendung die physikalischen Aussagen nicht ändern soll, d. h., daß sie mit den Meßoperatoren vertauschen; dasselbe gilt von $T^{2}$ und $\Pi^{2}$. Der Operator $T^{2}$, bzw. die Operatoren $\Pi^{2}$, besitzen je zwei Eigenräume, während die Operatoren $q, N, \mathbb{\Omega}$ unendlich viele haben.

Man sieht unmittelbar, daß für zwei Vektoren die relative Phase unbestimmt wird, wenn sie in zwei verschiedenen Eigenräumen der angegebenen Operatoren liegen. Der Zustandsraum der physikalischen Zustände zerfällt damit in kohärente Sektoren, wobei zwei Sektoren sich durch mindestens einen Eigenwert eines Operators $B_{k}$ unterscheiden.

Man kann auch Systeme $\psi$ beschreiben, die in zwei verschiedenen Sektoren von Null verschiedene Komponenten besitzen; es handelt sich dann nicht um einen Zustand oder genauer um einen reinen Zustand, sondern um ein Gemenge $\psi_{1}$ in $\widetilde{\Xi}_{1}{ }^{\prime \prime}, \psi_{2}$ in $\widetilde{\Xi}_{2}^{\prime \prime}$, das nach den Methoden der statistischen Mechanik zu behandeln ist.

Man schreibt $\psi=\left\{c_{1} \psi_{1}, c_{2}, \psi_{2}\right\}$, manchmal auch $\psi=c_{1} e^{i a_{1}} \psi_{1}+c_{2} e^{i a_{2}} \psi_{2}$, wobei am Ende über $\alpha_{1}$ und $a_{2}$ gemittelt wird.

Für einen der hier beschriebenen Sektoren der physikalischen Zustände ist der kohärente Sektor $\Im^{\prime \prime}$ aus Teil II, § 3, repräsentativ. Die Elemente von $\Im_{l}$, welche nicht in $\mathfrak{S}_{l}^{\prime \prime}$ liegen, gingen in die Betrachtungen dieses Paragraphen bis jetzt nicht ein. Sofern man aber das Transformationsverhalten unter den Operatoren $B_{k}$ auch für die nicht-physikalischen $\mathrm{Zu}$ stände festgelegt hat, tritt gleichzeitig mit dem Zer- 
fall des Zustandsraumes der physikalischen Zustände in kohärente Sektoren der Zerfall des gesamten $\mathrm{Zu}$ standsraumes in kohärente Sektoren ein. Über die zu $\widetilde{\Xi}_{l}$ und nicht zu $\widetilde{\Xi}_{l}^{\prime \prime}$ gehörenden Elemente kann man dann noch nach anderen Gesichtspunkten befinden, z. B. könnte die Menge dieser Elemente für bestimmte $\widetilde{\Xi}_{l}$ leer sein.

Über den Fundamentaltensor $g$ ist hinsichtlich des Zerfallens des Zustandsraumes in kohärente Sektoren folgendes zu bemerken: Die Untermatrizen von $g$, die simultan mit den Symmetriegruppen, welche die Sektoren $\widetilde{\Xi}_{l}$ invariant lassen, ausreduzieren, zerfallen auch gleichzeitig mit der Einteilung in Sektoren.

Wie in den vorangehenden Abschnitten ausführlich diskutirt, können aber auch irreduzible Darstellungen vorkommen, mit denen $g$ nicht gleichzeitig zerfällt. In diesem Falle ist dann auch denkbar, daß $g$ nicht gleichzeitig mit der Sektoreinteilung zerfällt.

Beispiel: Bei der Darstellung

$W=\left(\begin{array}{cc}\left\{V_{1}\right\} & 0 \\ 0 & \left\{\left(V_{1}^{*}\right)^{-1}\right\}\end{array}\right)$ mit $\left\{V_{1}\right\}$ nicht äquivalent zu $\left\{\left(V_{1}^{*}\right)^{-1}\right\}$ und dem Fundamentaltensor $g=\left(\begin{array}{cc}I & 0 \\ 0 & I\end{array}\right)$ können der obere der beiden Darstellungsräume $\{\varphi\}$ in einem Sektor $\widetilde{\subseteq}_{k}$, der untere $\{\varphi\}$ in einem Sektor $\Xi_{l}$ liegen.

\section{$\S 2$. Positive und negative g-Normen für die physikalischen Zustände}

Im folgenden kommt es nur noch auf die Teilräume $\widetilde{\Xi}_{k}^{\prime \prime}$ der kohärenten Sektoren an, in denen die physikalischen Zustände liegen. Diese besitzen definite Normen und man kann annehmen, daß es sich bei ihnen um Hilbert-Räume $\widetilde{\mathfrak{S}}_{k}$ handelt. In jedem induzieren die Transformationsgruppen, insbesondere die inhomogene Lorentz-Gruppe, unitäre Darstellungen $U_{k}$. Diese zerfallen in irreduzible unitäre Darstellungen $U_{n k}$. Für jede unitäre irreduzible Darstellung $U_{n k}$ wird der Fundamentaltensor ein Vielfaches der Einheitsmatrix, also $U_{n k}=\hat{\lambda}_{n k} I$. Damit die g-Normen selbst als Wahrscheinlichkeiten gedeutet werden können, müssen die $\lambda_{n k}$ positiv und reell sein, man kann also $\lambda_{n k}=1$ setzen.

Es ist jedoch möglich, auch andere Werte für $\lambda_{n k}$ zuzulassen, sofern man die Wahrscheinlichkeitsinterpretation etwas modifiziert.

Sei die Einheit $I_{k}$ des kohärenten Sektors $\widetilde{\subseteq}_{k}^{\prime \prime}$ in einen Satz von Projektionsoperatoren $P_{m}$, wie sie in $\S 1$ von Teil II eingeführt wurden, zerlegbar.

$$
I_{k}=\sum P_{m}
$$

Dabei ist nunmehr nach den einschränkenden Bedin- gungen von Teil II $\left\langle\varphi\left|P_{m}\right| \varphi\right\rangle$ nicht negativ. Wenn jedoch die Ausdrücke $\left\langle\varphi\left|P_{m}\right| \varphi\right\rangle=a_{m} e^{i \lambda}$ sind, wobei $a_{m}$ reell und nicht negativ, $\lambda$ reell und nicht von $m$ abhängig ist, so werden die Ausdrücke

$$
w_{m}=\frac{\left\langle\varphi\left|P_{m}\right| \varphi\right\rangle}{\langle\varphi \mid \varphi\rangle}
$$

reell und nicht negativ. Außerdem gilt

$$
\sum w_{m}=1 \text {. }
$$

Es läßt sich daher eine Größe $w_{m}$ als Wahrscheinlichkeit dafür interpretieren, das System bei der entsprechenden Messung in dem zu $P_{m}$ zugehörigen Teilraum anzutreffen.

Hieraus erkennt man, daß zunächst $\lambda_{n k}=e^{i \varrho_{k}}$ möglich ist, wobei man die Phase gleichmäßig für den ganzen kohärenten Sektor $\widetilde{\subseteq}_{k}^{\prime \prime}$ zu wählen hat. Bei Beschränkung auf Hermitesche Fundamentaltensoren wird diese Freiheit auf die Wahl des Vorzeichens eingeschränkt, so daß schließlich $g_{k}= \pm I_{k}$ sein kann.

Eine weitere Verallgemeinerung des Fundamentaltensors in der Richtung, daß man zu verschiedenen irreduziblen Darstellungen $U_{n k}$ ein und desselben Sektors für $\lambda_{n k}= \pm 1$ zuläßt, verbietet sich nach den Überlegungen von Teil II, § 3 .

Neben reinen Zuständen muß man auch Gemengє betrachten. Im Hinblick auf die Überauswahlregelr kann man zwei einfache Arten von Gemengen unterscheiden :

1. Die Gemenge, welche üblicherweise im Zusanımenhang mit dem Meßvorgang diskutiert werden und für deren Entstehen die oben angegebene Zerlegung nach Projektionsoperatoren charakteristisch ist: Ein physikalisches System läuft als reiner $\mathrm{Zu}$ stand in den Meßapparat ein; nach dem Meßprozeß ist diesem System, sofern man auf die Kenntnisnahme des Meßresultates verzichtet, im allgemeinen ein Gemenge zugeordnet. Dieser Vorgang spielt sich innerhalb eines kohärenten Sektors ab.

2. Die Gemenge, welche sich aus Zuständen $\left\{\varphi_{i}, \varphi_{k}\right\}$ zusammensetzen, die in verschiedenen kohärenten Sektoren $\mathscr{S}_{i}$ bzw. $\mathscr{S}_{k}$ liegen. $\varphi_{i}$ für sich und $\varphi_{k}$ für sich sollen reine Zustände sein. Diese zweite Art von Gemengen kann aus einem reinen Zustand niemals durch einen Meßprozeß entstehen. Ein reiner Zustand hat nur in einem einzigen kohärenten Sektor eine von Null verschiedene Komponente und eine Messung kann ihn wohl in ein Gemenge, aber nicht in eines der Art 2) überführen. 
Gemenge nach 2) können im Sinne der klassischen Statistik als Anfangszustand vorliegen. Am System durchgeführte Messungen ändern, sofern man auf die Kenntnisnahme der Meßresultate verzichtet, nichts an der ursprünglich vorliegenden Wahrscheinlichkeitsverteilung über die kohärenten Sektoren $\tilde{\mathfrak{Y}}_{k}$. Das folgt ja alles sofort aus der Vertauschbarkit der zu den Überauswahlregeln gehörenden Operatoren $B_{l}$ mit den Meßoperatoren.

Neben diesen zwei Arten von Gemengen können natürlich auch solche vorkommen, die gleichzeitig Gemenge im Sinne von 1) und im Sinne von 2) sind.

Während man sonst bei definiter Metrik für ein Gemenge $\left\{\varphi_{1}, \varphi_{2}, \ldots, \varphi_{n} \ldots\right\}$ für die Wahrscheinlichkeiten, daß der Zustand $\varphi_{1}, \varphi_{2}, \ldots, \varphi_{n}, \ldots$ vorliegt, $w_{n}=\frac{\left\langle\varphi_{n} \mid \varphi_{n}\right\rangle}{\sum_{l}\left\langle\varphi_{l} \mid \varphi_{l}\right\rangle}$ erhält, muß man hier bei Gemengen zweiter Art $w_{n}=\frac{\left|\left\langle\varphi_{n} \mid \varphi_{n}\right\rangle\right|}{\sum_{l}\left|\left\langle\varphi_{l} \mid \varphi_{l}\right\rangle\right|}$ ansetzen.

Es bleibt die Frage zu beantworten, ob man willkürlich für jeden der kohärenten Sektoren für $g_{k}=+I$ oder $g_{k}=-I$ wählen darf. Man überblickt den Sachverhalt am besten, wenn man als Basis in den Hilbert-Räumen $\mathfrak{S}_{k}$ die Zustände zu ein- oder auslaufenden Feldern im Sinne des L.S.Z.-Formalismus ${ }^{20}$ benutzt. Nach $\mathrm{H}_{\mathrm{AAG}}{ }^{21}$ ist die Norm von Systemen, die aus genügend weit voneinander entfernten Teilchen bestehen unter bestimmten Bedingungen das Produkt der Normen der Einteilchenzustände. Die Schwierigkeit, daß diese Bedingungen z. B. im Falle des elektromagnetischen Feldes nicht erfüllt sind, muß hier ausgeklammert werden. Es wird angenommen, daß die Norm stets gleich dem Produkt der Normen der ein- oder auslaufenden Einteilchenzustände ist.

Daraus folgt, daß die Freiheit $g_{k}=+\boldsymbol{I}$ oder $g_{k}=-I$ zu wählen nur für eine gewisse Basis in der Menge der kohärenten Sektoren $\mathfrak{S}_{k}$ besteht. Die Basis setzt sich aus $f$ kohärenten Sektoren $\mathfrak{H}_{k}$ zusammen, wenn es $f$ Überauswahlregeln gibt. Bei den aufgeführten vier Überauswahlregeln zu $T^{2}, Q, N, \Omega$ besteht sie aus einem kohärenten Sektor mit halbzahligem Spin, einem zweiten mit ungeradem $Q^{\prime}$, einem dritten mit ungeradem $N^{\prime}$ und einem vierten mit ungeradem $\Omega^{\prime}$, wobei die gestrichenen Größen die Eigenwerte zu den entsprechenden Operatoren sind. Nimmt man $\Sigma_{k}{ }^{\prime}$ ganz- oder halbzahlig, je nachdem ob der Sektor $\Im_{k}$ zu ganz- bzw. halbzahligen Spinzuständen gehört, so hat man z. B. die Möglich- keit, den metrischen Fundamentaltensor

$$
g_{k}=(-I) \exp \left[Q_{k}{ }^{\prime}+N_{k}{ }^{\prime}+\mathfrak{Q}_{k}{ }^{\prime}+2 \Sigma_{k}{ }^{\prime}\right]
$$

zu wählen oder allgemein die Möglichkeiten

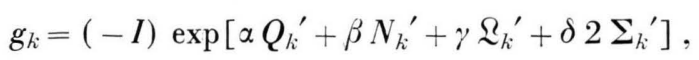

wobei $\alpha$ gleich 0 oder $1, \beta$ gleich 0 oder $1, \ldots$, $\delta$ gleich 0 oder 1 gewählt werden können.

\section{§3. Meßvorgang an physikalischen Systemen mit indefiniter Norm}

Im vorigen Abschnitt wurde formal begründet, daß man auch für physikalische Zustände positive und negative $g$-Normen verwenden kann. Diese Freiheit ist durch den Zerfall des Zustandsraumes in kohärente Sektoren gewährleistet; der Zerfall gründet sich auf die Existenz gewisser Operatoren $B_{k}$, die mit allen Meßoperatoren $A_{m}$ vertauschen. Da alle möglichen Meßoperatoren auf alle denkbaren physikalischen Systeme angewandt eine schlecht überschaubare Gesamtheit von Meßvorgängen darstellt, sollen die für die Zerfällung wesentlichen Elemente bei einem Meßvorgang diskutiert werden. Dabei mag sich das Meßobjekt vor Eintritt in den Meßapparat in einem reinen Zustand $|\psi\rangle$ befinden. Der Meßapparat liegt im allgemeinen als Gemenge vor. Daß der Anfangszustand des Apparates kein reiner Zustand ist, hat jedoch auf die wesentlichen Punkte des folgenden Gedankenganges keinen Einfluß ${ }^{22}$. Es soll daher zur Vereinfachung angenommen werden, daß auch der Anfangszustand des Apparates ein reiner Zustand $|\Psi\rangle$ ist. Bei der Erörterung des Meßvorganges geht man durch die „Erweiterung“ zum Zustand Meßobjekt + Meßapparat über, der durch $\Phi=|\Psi\rangle \times|\psi\rangle$ beschrieben werden soll. Wir benutzen das Heisenberg-Bild. Als Basis ist zunächst die der einlaufenden Zustände verwandt. Bei Wahl dieser Basis wird das Transformationsverhalten unter den Symmetriegruppen offensichtlich: Es treten die direkten Produkte der den Transformationen im Darstellungsraum der $\{\Psi\}$ bzw. im Darstellungsraum $\{\psi\}$ zugeordneten Matrizen auf.

Eine Basis für die auslaufenden Zustände des Meßapparates heiße $\Pi_{j}$, die des Meßobjektes $\pi_{k}$.

20 H. Lehmann, K. Symanzik u. W. Zimmermann, Nuovo Cim., I, Serie X, 205 [1955].

21 R. HAaG, Vortrag gehalten auf der intern. Konferenz in Lille (1957).

22 G. Süssmans, Bayer. Akad. d. Wissensch. Math.-Naturws. Klasse, Neue Folge, Heft 88. 
Der Meßvorgang sorgt für eine eindeutige Zuordnung der $j$ und $k$, so $\operatorname{da} \beta \Phi=\sum_{j} \Phi^{j}\left|\Pi_{j}\right\rangle \times\left|\pi_{j}\right\rangle$ wird. Um die Übereinstimmungswahrscheinlichkeiten bestimmen zu können, muß man die Metrik festlegen: Sei etwa

$$
g_{k}=(-I) \exp \left[Q_{k}{ }^{\prime}+N_{k}{ }^{\prime}+\Omega_{k}{ }^{\prime}+2 \Sigma_{k}{ }^{\prime}\right]
$$

gewählt, so daß z.B. ein einlaufendes Proton $|\psi\rangle$ die $g$-Norm -1 erhält. Nehmen wir für $|\Psi\rangle$ an, es befinde sich in einem Sektor $\mathscr{S}_{k}$ mit der $g$-Norm +1 ! Dann wird

$$
\langle\Phi \mid \Phi\rangle=\langle\Psi \mid \Psi\rangle\langle\psi \mid \psi\rangle=+1(-1)=-1 .
$$

Von den $\left|\Pi_{j}\right\rangle$ soll angenommen werden, daß es sich um orthogonale Zustände handelt, d. h. der Meßapparat muß zweckmäßig so gebaut sein, daß man aus den Messungen eindeutig auf das Vorliegen eines $\left|\pi_{k}\right\rangle$ und auf das Nichtvorliegen aller anderen $\left|\Pi_{j}\right\rangle, j \neq k$, schließen kann. Im allgemeinen steckt in dieser Forderung eine starke Idealisierung; daß die meisten Meßvorgänge dieser nicht nahe kommen, ist hier für die Diskussion nicht wesentlich. Mit dem Projektionsoperator $P_{\mathrm{m}}$, der im Falle des Produktzustandes hier lautet

$$
P_{m}=\left|\Pi_{m}\right\rangle \times\left|\pi_{m}\right\rangle\left\langle\pi^{m}\right| \times\left\langle\Pi^{m}\right|
$$

(über $m$ nicht summieren!)

ergibt sich $\left\langle\Phi\left|P_{m}\right| \Phi\right\rangle=\Phi_{m}{ }^{*} \Phi^{m}=-\left|\Phi^{m}\right|^{2}$.

Man sieht, daß mit

$$
w_{m}=\frac{\Phi_{m}^{*} \Phi^{m}}{\langle\Phi \mid \Phi\rangle}=\frac{-\left|\Phi^{m}\right|^{2}}{\Sigma \Phi^{*} \Phi^{l}}=\frac{-\left|\Phi^{m}\right|^{2}}{-\Sigma\left|\Phi^{l}\right|^{2}}
$$

als Teilwahrscheinlichkeiten bis jetzt keine Schwierigkeiten mit der Wahrscheinlichkeitsinterpretation auftreten. Bei der Theorie des Meßprozesses verlangt man aber, daß man durch einen Schnitt den Meßapparat abtrennen und die Wahrscheinlichkeitsinterpretation für den Zustand $\psi$ des Meßobjektes allein durchführen kann. Hierzu ist notwendig, daß $\psi$ und $\pi_{m}$ in kohärenten Sektoren $\mathscr{S}_{k}$ mit demselben Vorzeichen von $\langle\chi \mid \chi\rangle$ liegen, sofern sich $\chi$ in $\mathscr{\mathfrak { S }}_{k}$ befindet.

An dieser Stelle wird die Bedeutung der Überauswahlregel sichtbar: Sie sorgt dafür, daß Meßapparat für sich und Meßobjekt für sich bei der Wechselwirkung zwischen Apparat und Objekt in dem gleichen kohärenten Sektor verbleiben, so daß $\Psi$ und $\Pi_{m}$ simultan in einem Sektor $\mathscr{S}_{l}$ und ebenfalls $\psi$ und $\pi_{m}$ simultan in einem Sektor $\mathfrak{S}_{k}$ liegen, wobei im allgemeinen $l \neq k$.
Für die vier angegebenen Überauswahlregeln bedeutet das physikalisch, daß bei einer Messung vom Meßapparat auf das Meßobjekt (oder umgekehrt) kein halbzahliger Spin, keine elektrische Ladung, keine Baryonladung, keine Leptonladung übertragen werden dürfen. Solange beim Meßvorgang irgendein elektrisches Feld für die Wechselwirkung verantwortlich ist, sind diese Bedingungen wegen der Eigenschaft dieses Feldes, neutral hinsichtlich der drei Ladungsarten zu sein, und der Eigenschaft der Photonen, einen ganzzahligen Spin zu tragen, erfüllt. Im umgekehrten Fall, wenn ein physikalisches System A mit einem anderen System B in eine solche Wechselwirkung tritt, daß z. B. Ladung ausgetauscht wird, so kann man diesen Vorgang nicht mehr eine Messung am System A im oben angegebenen Sinne nennen. Dann versagt auch schon bei Verwendung einer definiten Metrik die Wahrscheinlichkeitsinterpretation, wenn man durch einen Schnitt den Meßapparat B abtrennt.

\section{$\S 4$. Abbildungen von kohärenten Sektoren auf andere kohärente Sektoren}

Es seien am Anfang einige Gruppen genannt, mit deren Transformationen Abbildungen von einem kohärenten Sektor des Zustandsraumes auf einen anderen verknüpft sind, z. B. die drei Gruppen, von denen jeweils jede zwei Elemente besitzt, erstens I, CPT, zweitens I, CP, drittens I, C, wobei C die Ladungskonjugation, $\mathrm{P}$ die Paritätsoperation und $\mathrm{T}$ die Zeitumkehroperation bedeuten. Die letzte dieser Transformationsgruppen gilt nach den neueren Ergebnissen zwar nicht mehr in Strenge als Symmetriegruppe, kann aber immerhin in idealisierten $\mathrm{Zu}$ standsräumen, in denen von den schwachen Wechselwirkungen abgesehen ist, als Symmetriegruppe verwendet werden. Die drei Gruppen besitzen die gemeinsame Eigenschaft, daß ihr von I verschiedenes Element einen kohärenten Sektor mit den Quantenzahlen $Q^{\prime}, N^{\prime}, \mathfrak{R}^{\prime}$ auf einen mit den Quantenzahlen $-Q^{\prime},-N^{\prime},-\mathbb{R}^{\prime}$ abbildet. Da sich bei dieser Abbildung die Quantenzahlen um eine gerade Anzahl ändern, andererseits der Spin (gemeint ist dabei nicht die Richtung des Spins, sondern die Zahl $s(s+1)$ ] erhalten bleibt, geht auch bei Verwendung von positiven und negativen Normen für physikalische Zustände nach S. 556 ein Zustand positiver $g$-Norm wieder in einen solchen über, ebenso ein Zustand negativer $g$-Norm in einen mit negativer $g$-Norm. Die den Gruppenelementen zugeordneten 
Transformationen können also weiterhin unitär sein.

Betrachtet man dagegen die Transformationen, die mit der Isospingruppe verknüpft sind, so ändert sich dieser Sachverhalt. Diese Gruppe ist ja der dreidimensionalen Drehgruppe isomorph. Bezeichnet man die drei infinitesimalen Elemente mit $J_{x}, J_{y}, J_{z}$ und setzt das Polynom $J_{x}{ }^{2}+J_{y}{ }^{2}+J_{z}{ }^{2}=J^{2}$, so richtet man es für gewöhnlich so ein, daß $J_{z}$ einen kohärenten Sektor auf sich selbst abbildet, während der Operator $J_{x}+i J_{y}$ innerhalb eines zum Eigenwert $j(j+1)$ des Operators $J^{2}$ gehörigen Multipletts jede Komponente des Multipletts in die nächste mit einer um 1 höheren Ladung, die Komponente mit der höchsten Ladung jedoch auf Null abbildet. Damit wird, sofern man in der Formel S. $557 \alpha=1$ setzt, eine Abbildung induziert, welche Zustände mit positiver $g$-Norm in Zustände mit negativer $g$-Norm (und umgekehrt) überführt. Die der Isospingruppe zugeordneten Transformationen im Zustandsraum sind dann weder unitär, noch erhalten sie die $g$ Norm. Trotzdem gelang die Wahrscheinlichkeitsinterpretation. Das hat seinen Grund darin, daß den infinitesimalen Operatoren der Isospingruppe im allgemeinen keine meßbaren Operatoren zugeordnet sind. Dagegen gehört der Operator $J_{z}$, der mit der Ladung des Systems verknüpft wird, wieder zu einer meßbaren Größe.

Üblicherweise findet das Vorhandensein von Symmetriegruppen seinen Niederschlag in zugeordneten unitären Transformationen im HiLbert-Raum. Es ist deshalb vielleicht zweckmäßig, an einem einfachen Beispiel anzugeben, wie man das Prinzip der Ladungsunabhängligkeit der mesonischen Kräfte in einer etwas anderen Weise formulieren kann. Man vermeidet dann noch einen anderen Nachteil, der mit der üblichen Formulierung zusammenhängt: Wenn man jedem Element der Isogruppe eine Transformation im Zustandsraum zuordnet, dann wird im allgemeinen ein Vektor, der ganz innerhalb eines kohärenten Sektors liegt, in einen übergeführt, der in verschiedenen Sektoren von Null verschiedene Komponenten besitzt, d. h. ein reiner Zustand wird in ein Gemenge 2. Art (S. 556) abgebildet. Man faßt daher im allgemeinen stillschweigend nur solche Transformationen ins Auge, welche bestimmten Elementen der Drehgruppe bzw. des Infinitesimalrings der Drehgruppe zugeordnet sind und bei denen ein Vektor aus einem kohärenten Sektor in einen abgebildet wird, der wieder ganz innerhalb eines anderen kohärenten Sektors liegt. In Wahrheit ist man dann also bei den Transformationen im Hilbert-Raum von einer kontinuierlichen Transformationsgruppe zu einer diskreten übergegangen.

\section{§ 5. Beispiel: Prinzip der Ladungsunabhängigkeit der Kernkräfte in einem einfachen Fall *}

Wie man das Prinzip der Ladungsunabhängigkeit mit Hilfe diskreter Operationen, welche einen kohärenten Sektor auf einen anderen abbilden, formulieren kann, soll im folgenden für das a-Nukleonproblem gezeigt werden. Dabei ist an solche Verhältnisse gedacht, bei denen man in guter Näherung die Wirkung des Mesonenfeldes durch ein klassisches Potentialfeld beschreibt. Im übrigen ist es gleichgültig, ob es sich um ein Streuproblem oder um einen stabilen Kern handelt. Der Einfluß des die Symmetriegruppe störenden CouLomb-Feldes wird weggelassen und im Einklang damit auch der Massenunterschied zwischen Proton und Neutron.

Seien die mit dem so formulierten a-Nukleonenproblem speziell auftretenden kohärenten Sektoren mit $\mathscr{S}_{a}{ }^{0}, \mathscr{\mathfrak { F }}_{a}{ }^{1}, \ldots, \mathfrak{S}_{a}{ }^{a}$ bezeichnet, wobei der obere Index die Zahl der Protonen, also die Zahl der Elementarladungen, die für den Sektor charakteristisch ist, angibt.

Gäbe es kein PAULI-Prinzip, so könnte man das Prinzip der Ladungsunabhängigkeit der Kernkräfte etwa so formulieren: $\mathrm{Zu}$ jedem physikalischen System, dem ein Vektor in $\mathfrak{S}_{a}{ }^{k}$ zugeordnet ist, gibt es weitere Systeme, die sich untereinander und zum ersten gleich verhalten, von deren zugeordneten Vektoren je einer in jedem anderen Sektor $\mathfrak{S}_{a}{ }^{l}, l \neq k$, liegt. Gleich verhalten soll heißen, daß die Meßresultate von allen möglichen Messungen (Messungen, die elektromagnetische Eigenschaften betreffen, sind sinngemäß auszunehmen) an zwei dermaßen zugeordneten Systemen übereinstimmen.

Zwei in diesem Sinne gleiche Systeme, die in zwei verschiedenen Sektoren liegen, sollen aufeinander abgebildet und durch diese Abbildung ihre Gleichheit zum Ausdruck gebracht werden.

Infolge des Pauli-Prinzips, das den Zustandsraum in den verschiedenen Sektoren verschieden stark einschränkt, - z. B. werden im Falle $a=2$ wohl $\mathfrak{F}_{2}{ }^{0}$ und $\mathfrak{S}_{2}{ }^{2}$, aber nicht ${\mathfrak{\mathfrak { Y } _ { 2 }}}^{1}$ eingeschränkt - ,

* Für die Anregung, die Symmetrieeigenschaft der Ladungsunabhängigkeit der Mesonkräfte unmittelbar mit Hilfe einer diskreten Gruppe von Abbildungen im Zustandsraum zu formulieren sowie für Diskussionen über diesen Gegenstand, bin ich Herrn Prof. HAag zu Dank verpflichtet. 
ist die oben aufgestellte Formulierung des Prinzips der Ladungsunabhängigkeit nicht richtig. Es muß heißen: Zu jedem physikalischen System mit einem Vektor in dem Sektor $\mathfrak{H}_{a}{ }^{k}$ gibt es ein gleiches mit einem Vektor in dem Sektor $\mathscr{H}_{a}^{l}$, sofern das nach dem Pauli-Prinzip erlaubt ist.

Die Übersicht darüber, wie die Abbildbarkeit durch das Pauli-Prinzip eingeschränkt wird, gewinnt man durch Zerlegung der einzelnen Sektoren nach irreduziblen Darstellungen der symmetrischen Gruppe. Hier sollen der Gedankengang der Methode und die Ergebnisse nach den verschiedenen Abschnitten mitgeteilt werden. Die Einzelheiten und Beweise sind im Anhang niedergelegt; ähnliche Verfahren wurden bereits in anderem Zusammenhang schon früher in der Quantenmechanik angewandt ${ }^{23}$.

Der I. Schritt bringt die mathematische Methode,

der II. die Zerlegung eines Zustands $\Psi$ nach irreduziblen Darstellungen der Permutationsgruppe,

der III. die Abbildung der kohärenten Sektoren aufeinander,

der IV. den Zusammenhang mit dem Isotopenspinformalismus.

I. Mathematische Methode: Die irreduziblen Darstellungen der Symmetriegruppe $\Im_{a}$ (Gruppe der $a$ ! Permutationen von $a$ Elementen) werden durch Zerlegung der regulären Darstellung gewonnen. Dabei kommt es auf die Zerlegung nach Klassen äquivalenter Darstellungen an. Diese geschieht durch eine Zerlegung der I nach Erzeugenden einfacher zweiseitiger Ideale. Bei der Gewinnung dieser Erzeugenden spielen die Young-Tableaux eine Rolle. Diese bestehen aus Rahmen mit $a$ Kästchen, in welchen die $a$ Zahlen der Symmetriegruppe $\widetilde{\Im}_{a}$ eingetragen sind. Die Zeilenlänge der Rahmen nimmt von oben nach unten und die Spaltenlänge von links nach rechts ab. Es gibt z. B. zu $a=4$ die folgenden Möglichkeiten :

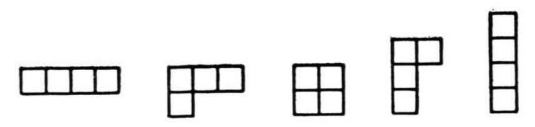

Abb. 1.

Für die folgende Darlegung ist wesentlich, daß jeder Klasse äquivalenter irreduzibler Darstellungen der Gruppe $\widetilde{\Im}_{a}$ ein bestimmter Rahmen zugeordnet ist. Es gibt also im Falle $a=4$ fünf solcher Klassen. Jede Klasse soll durch den zugeordneten Rahmen

23 F. Hund, Z. Phys. 43, 788 [1927]. symbolisiert werden. Im Zusammenhang mit dem Pauli-Prinzip wird ein Satz über assoziierte irreduzible Darstellungen gebraucht. Die assoziierte Darstellung gehört zu einem Rahmen, dessen Zeilenlängen gleich den Spaltenlängen des ursprünglichen Rahmens sind. Es sind oben z. B. die irreduziblen Darstellungen zum Rahmen

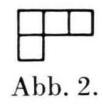

und die zum Rahmen

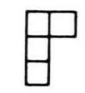

Abb. 3 .

assoziiert.

Es gilt für zwei irreduzible Darstellungen $\Delta$ und $\Delta^{\prime}$ :

Reduziert man das Produkt $\Gamma=\Delta \times \Delta^{\prime}$ aus, so tritt in $\Gamma$ die antimetrische Darstellung dann und nur dann auf, und zwar genau einmal, wenn $\Delta$ und $\Delta^{\prime}$ assoziiert sind.

II. Sei $\psi$ ein Zustand mit $n$ Protonen und $a-n$ Neutronen

$$
\psi=\psi\left(x_{1}, \ldots, x_{n} \mid x_{n+1}, \ldots, x_{a}\right) .
$$

Dieser gehorcht dem Pauli-Prinzip, ist also totalantimetrisch unter den Permutationen der Untergruppe $\widetilde{\Im}_{\langle 1, \ldots, n\rangle\langle n+1, \ldots, a\rangle}$ der Gruppe $\widetilde{\Xi}_{a}$. Bei $\widetilde{\mathfrak{S}}_{\langle 1, \ldots, n\rangle\langle n+1, \ldots, a\rangle}$ sind Permutationen von $1, \ldots, n$ unter sich und von $n+1, \ldots, a$ unter sich zugelassen. $x_{i}$ bedeutet einen Satz von drei Ortskoordinaten und einer Spinkoordinate. Ohne Beeinträchtigung der Allgemeinheit kann man $n \geqq a-\mathrm{n}$ annehmen.

Für die Zerlegung von $\psi$ nach irreduziblen Darstellungen von $\widetilde{\Im}_{a}$ gilt folgendes:

1. Es kommen nur solche irreduziblen Darstellungen vor, deren zugehörige Rahmen nicht mehr als zwei Spalten besitzen und die in der ersten Spalte mindestens $n$ Kästchen haben.

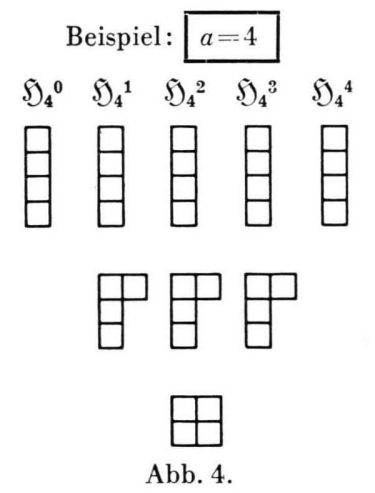


2. Wenn $\psi$ keine anderen Symmetrieeigenschaften als die der totalen Antimetrie unter $\widetilde{\Xi}_{\langle 1, \ldots, n \times n+1, \ldots, a\rangle}$ besitzt, so kommt jede der nach 1 . erlaubten irreduziblen Darstellungen genau einmal vor.

III. In jeder der genau je einmal vorkommenden erlaubten irreduziblen Darstellungen von $\Im_{a}$ werden die unter $\widetilde{\Xi}_{\langle 1, \ldots, n\rangle\langle n+1, \ldots, a\rangle}$ total antimetrischen Vektoren aufgesucht: Nach einem Reziprozitätstheorem von Frobenius (s. Anhang), das Aussagen über die Häufigkeit von vorkommenden irreduziblen Darstellungen macht, kann man zeigen: In jeder der erlaubten irreduziblen Darstellungen von $\Im_{a}$ kommt ein unter $\widetilde{\Xi}_{\langle 1, \ldots, n\rangle\langle n+1, \ldots, a\rangle}$ total-antimetrischer Vektor genau einmal vor.

Ausgehend von $\psi\left(x_{1}, \ldots, x_{n} \mid x_{n+1}, \ldots, x_{a}\right)$, das total antimetrisch unter $\Im_{\langle 1, \ldots, n \times\langle n+1, \ldots, a\rangle}$ ist, also dem Pauli-Prinzip gehorcht, kann man also durch Anwendung von Permutationsoperatoren und Linearkombinationen zu jeder erlaubten irreduziblen Darstellung $\Delta_{l}$ ein und nur ein $\psi_{\Delta_{l}}$ (ein komplexer Faktor bleibt natürlich frei, der durch Normierung festzulegen ist) erzeugen, das dem Darstellungsraum von $\Delta_{l}$ angehört.

Die Abbildung besteht darin, daß die erhaltenen Vektoren $\left(\psi_{\Delta l}\right)_{a}{ }^{k}$ mit festem $l$ und laufendem $k$ einander zugeordnet werden und so die Gleichheit der repräsentierten physikalischen Systeme ausgesprochen ist.

IV. Beispiel und Zusamenhang mit dem Isotopenspinformalismus:

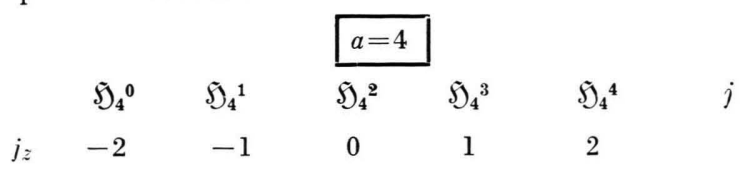
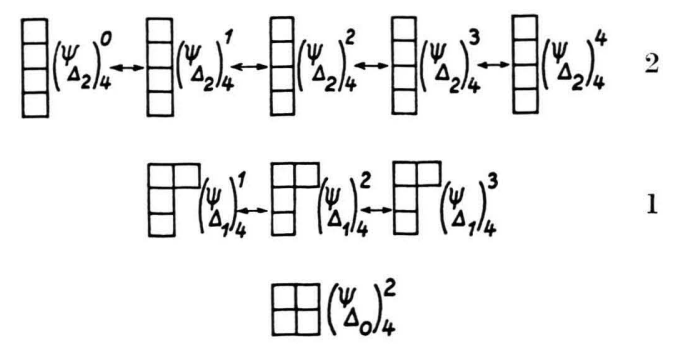

Abb. 5 .

$\mathrm{Zu}$ jeder der durch den Rahmen symbolisierten irreduziblen Darstellungen von $\Im_{a}$ gibt es nach oben genau einen unter $\Im_{<1, \ldots, n \times n n+1, \ldots, a\rangle}$ total antimetrischen Zustand, der rechts neben den zugehörigen Rahmen geschrieben ist. Die Abbildung ist durch
Pfeile gekennzeichnet. Unten sind die Eigenwerte von $J_{z}$, rechts der erste Faktor vom Eigenwert $j(j+1)$ zum Eigenwert von $J^{2}$ angegeben, die man bei Gebrauch des Isotopenspinformalismus den betreffenden Vektoren zuordnen würde. Wie der Zusammenhang zwischen dem hier eingeführten und dem Isotopenspinformalismus zustande kommt, ist im Anhang durchgeführt.

\section{$\S 6$. Eine mögliche Verallgemeinerung des „dualen" Zustandes}

Im letzten Abschnitt soll eine mögliche Verallgemeinerung, die weitgehend unabhängig von dem in den anderen Abschnitten Gesagten ist, erwähnt werden. Sie läuft darauf hinaus, daß man unter Umständen nicht unitäre Darstellungen auch in den Unterräumen der physikalischen Zustände verwendet. Hierzu muß man zu einer allgemeineren Definition des dualen Vektors übergehen. Von den invarianten Bilinearformen $\langle\Psi \mid X\rangle=(\Psi, g, X)=\Psi^{l^{*}} g_{l m} X^{m}$ wurde bisher dem Element die Bilinearform $\langle\Psi \mid \Psi\rangle=\Psi, g \Psi)$ als $g$-Norm zugeordnet, und hieran die Wahrscheinlichkeitsinterpretation geheftet. Etwas abstrakter gefaßt, führt man dabei den folgenden Prozeß durch: Dem Element $\Psi$ in $\mathscr{\mathfrak { S }}$ wird ein Elementenpaar $\Psi ; \Psi$ im Produktraum $\mathfrak{S} \times \mathfrak{S}$ zugeordnet. Diese Elementenpaare werden nach der Vorschrift $\Psi^{l^{*}} g_{l m} \Psi^{m}$ in den Körper der reellen Zahlen abgebildet. Die Verallgemeinerung für die Definition des dualen Elementes besteht nun darin, daß man zu $\Psi$ als duales Element nicht $\Psi$ wählt, sondern im ersten Faktorraum eine feste lineare Transformation vorgibt und das Paar $\mathfrak{Z} ; \Psi$ für die $g$-Norm-Bildung verwendet. Mit $\mathfrak{Q}^{i}{ }_{k}$ als Matrixelementen von $\mathfrak{Q}$ erhält man dann

für die $g$-Norm

$$
\left(\mathbb{L}_{k}^{i} \Psi^{k}\right)^{*} g_{i m} \Psi^{m},
$$

allgemein für Bilinearformen

$$
\left(\mathfrak{Q}^{i}{ }_{k} X^{k}\right)^{*} g_{i m} \Psi^{m} .
$$

Die Invarianz der Bilinearformen wird dadurch nicht beeinträchtigt. Das erkennt man wohl am besten in der Form

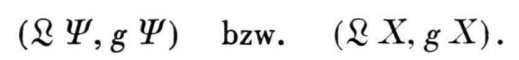

Bei einer Matrix $V(d)$, die das Gruppenelement $d$ darstellt, erhält man

$\left(\Omega \Psi, V^{*}(d) g V(d) \Psi\right)$ bzw. ( $\left(X, V^{*}(d) g V(d) X\right)$ 
und wegen $V^{*} g V=g$ ist das natürlich mit den oberen beiden Bilinearformen identisch. Wählt man z. B. $\mathfrak{Q}=g^{-1}$, so wird $\left(g^{-1} \Psi, g \Psi\right)$ positiv definit, da $g^{-1}=\left(g^{-1}\right)^{*}$.

Sinnvoll kann diese Verallgemeinerung nur auf solche Darstellungen angewandt werden, bei denen die Meßoperatoren Hermitesch sind. Dafür mag als Beispiel die von Dürr, Heisenberg u. a. ${ }^{24}$ angegebene Dehnungstransformation dienen. Die Dehnung $x_{\mu} \rightarrow a x_{\mu}$ im Ortsraum induziert im Zustandsraum eine bestimmte Darstellung. Da es sich um eine Abelsche Gruppe handelt, sind die irreduziblen Darstellungen eindimensional. Die Zustände transformieren sich dabei so: $\psi_{A_{n}^{\prime}} \rightarrow a^{\Lambda_{n}^{\prime}} \psi_{\Lambda_{n}^{\prime}}$, wobei $\Lambda_{n}{ }^{\prime}$ ein reeller Eigenwert zum infinitesimalen Operator $A$ für $\psi_{\Lambda_{n}^{\prime}}$ bei der Transformation kennzeichnend ist. Der Zustandsraum soll aber zu jedem $\psi_{A_{n}^{\prime}}$ auch ein $\psi_{-\Lambda_{n}^{\prime}}$ enthalten, das sich wie $\psi_{-\Lambda_{n}^{\prime}} \rightarrow a^{-\Lambda_{n}^{\prime}} \psi_{-\Lambda_{n}^{\prime}}$ transformiert. Der Fundamentaltensor ist analog dem, der unter Teil I, $\S 4 \mathrm{~b}$, für die inhomogene Lonentz-Gruppe angegeben wurde, d. h. $g=\left(\begin{array}{ll}0 & 1 \\ 1 & 0\end{array}\right)$ Dabei ist $\Psi_{\Lambda_{n}^{\prime}}$ z. B. als erster, $\Psi_{-\Lambda_{n}^{\prime}}$ als zweiter Vektor zu schreiben: $\Psi_{A_{n}^{\prime}}=\left(\begin{array}{l}1 \\ 0\end{array}\right), \quad \stackrel{\Psi}{\Psi}_{-\Lambda_{n}^{\prime}}=\left(\begin{array}{l}0 \\ 1\end{array}\right)$. Bliebe man bei der konventionellen Definition des dualen Vektors, so wäre , $\left.\Psi_{A_{n}^{\prime}}\right)$ das duale $\left(\Psi_{A_{n}^{\prime}}\right.$, zugeordnet. Es ergibt sich $\left(\psi_{A_{n}^{\prime}}, g \psi_{A_{n}^{\prime}}\right)=0$ und entsprechend $\left(\Psi_{-\Lambda_{n}^{\prime}}, g \Psi_{-\Lambda_{n}^{\prime}}\right)$. Dagegen wird bei der verallgemeinerten Zuordnung des dualen Vektors: ,$\left.\Psi_{A_{n}^{\prime}}\right)$ zugeordnet zu $\left(g^{-1} \Psi_{A_{n}^{\prime}},\right.$. Man erhält nunmehr für die verallgemeinerten Normen

$\left(g^{-1} \Psi_{\Lambda_{n}^{\prime}}, g \Psi_{\Lambda_{n}^{\prime}}\right)=1$ und $\left(g^{-1} \Psi_{-A_{n}^{\prime}}, g \Psi_{-\Lambda_{n}^{\prime}}\right)=1$.

Das heißt $\Psi_{\Lambda_{n}^{\prime}}$ bzw. $\Psi_{-\Lambda_{n}^{\prime}}$ sind auf 1 normiert, und zwar in invarianter Weise.

Hier sind aus dem Zustandsraum zwei der durch den Fundamentaltensor verbundenen Zustände herausgegriffen. Mag das Spektrum von $A$ diskret sein, und zwar $\Lambda_{n}{ }^{\prime}= \pm \frac{1}{2}, \pm \frac{3}{2}, \pm \frac{5}{2}, \ldots$ und ordnet man die Zustände z. B. von der Mitte aus nach oben mit wachsendem, nach unten mit abnehmendem Eigenwert zu $\Lambda$ an

$$
\Psi_{\frac{1}{2}}=\left(\begin{array}{c}
\vdots \\
\vdots \\
0 \\
1 \\
0 \\
0 \\
\vdots
\end{array}\right), \quad \Psi_{\frac{3}{2}}=\left(\begin{array}{c}
0 \\
0 \\
1 \\
0 \\
0 \\
\vdots \\
\vdots
\end{array}\right),
$$

$$
\ldots, \Psi_{-\frac{1}{2}}=\left(\begin{array}{c}
\cdot \\
\vdots \\
0 \\
1 \\
0 \\
\vdots \\
l
\end{array}\right), \quad \Psi_{-\frac{3}{2}}=\left(\begin{array}{c}
\cdot \\
\vdots \\
0 \\
0 \\
1 \\
\vdots \\
\vdots
\end{array}\right) \text {, }
$$

so erhält man den Fundamentaltensor

$$
g=\left(\begin{array}{c}
0 \\
.{ }^{1} 1^{1}{ }^{1} \\
.
\end{array}\right)
$$

Natürlich ist dieser Fundamentaltensor wegen der Entartung der Unterräume $\left\{\Psi_{A_{n}^{\prime}}\right\}$ noch zu gedrängt geschrieben. In Wahrheit hat man sich für jede 1 eine Untermatrix zu denken, die für die Invarianz der Bilinearformen unter den anderen Symmetriegruppen sorgt.

Für die Anregung zu dieser Arbeit und zahlreiche Diskussionen, besonders zum zweiten und dritten Teil, bin ich Herrn Prof. Heisenberg zu tiefem Dank verpflichtet. Herrn Prof. MAAK danke ich für kritische Bemerkungen zum ersten Teil, Herrn Prof. HaAg für die Anregung und Diskussionen zu einem Abschnitt des dritten Teiles, Herrn Dr. Symanzik für eine Diskussion zum ersten Teil, Herrn Dr. Dürr für eine kritische Durchsicht des ersten Teiles.

Der Max-Planck-Gesellschaft und der D e u t s c he n Forschungsgemeinschaft danke ich für die Gewährung eines Stipendiums.

\section{Anhang zum Prinzip der Ladungsunabhängigkeit der Kernkräfte}

\section{a) Häufigkeit der erlaubten irreduziblen Darstellungen ${ }^{25}$}

Man kann sich die Zustände durch die Projektionsoperatoren $e_{k}$ zerlegt denken:

$$
e_{k} \psi=\psi_{k} \text {. }
$$

Beachtet man, daß $e_{k}$ eine Summe von primitiven Idempotenten ist, $e_{k}=\sum_{k} e_{k i}$, welche zu den betreffenden Standardtableaux gehören, so scheint eine weitere Zerlegung der $\psi_{k}$ in $\psi_{k i}$ notwendig. $\widetilde{\Xi}_{a}$ angewandt auf $\psi_{k i}$ führt bei gleichem $k$ zu äquivalenten Darstellungen der Permutationsgruppe. Es wird als erstes bewiesen, daß sich diese äquivalenten Darstellungen auf eine reduzieren lassen. Falls man nicht zwischen äquivalenten irreduziblen Darstellungen unterscheidet, heißt das kurz: Jede

24 H.-P. Dürr, W. Heisenberg u. a., Z. Naturforschg. 14 a, 441 [1959].

25 Vgl. für die Bezeichnungen z. B. H. Boerner, Darstellungen von Gruppen, Springer-Verlag, Berlin 1955. 
irreduzible Darstellung kann höchstens einmal auftreten.

Beweis: Es ist $\psi_{k}=e_{k} \psi=e_{k} e_{\langle 1, \ldots, n\rangle}^{-} e_{\langle n+1, \ldots, a\rangle}^{-} \psi$ mit $e_{\langle i, \ldots, k\rangle}^{-}=\Sigma \pm p_{l}$ (summiert wird über alle Permutationen der Elemente $i, \ldots, k$, wobei + bei geraden, - bei ungeraden Permutationen steht). Der für $e_{k}$ repräsentative Rahmen kann höchstens zwei Spalten besitzen, deren erste mehr als $n$ Kästchen hat. In

$$
\psi_{k}=e_{\langle 1, \ldots, n\rangle}^{-} e_{\langle n+1, \ldots, a\rangle}^{-} \sum_{j} e_{k j} \psi
$$

tragen nur die $r$ Idempotente, deren Tableaux $1,2,3, \ldots, n$ in der ersten Spalte besitzen, etwas bei.

$$
\psi_{k}=e_{\langle 1, \ldots, n\rangle}^{-} e_{\langle n+1, \ldots, a\rangle}^{-} \sum_{j=1}^{+} e_{k j} \psi .
$$

Da es sich um Standardtableaux handelt, sehen sie so aus:

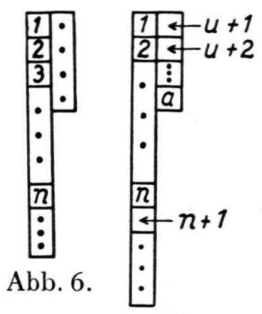

Abb. 7.
Die $r$ Standardtableaux können sich also nur durch eine Permutation der $n+1, n+2, \ldots, a$ unter sich unterscheiden.

Sei $T_{1}$ das Tableau, das rechts abgebildet ist, in dem die Zahlen, wenn man zuerst die erste Spalte, dann die zweite liest, in der natürlichen Reihenfolge stehen.

Sei $T_{i}=p_{i} T_{1}$, so gilt für die zugehörigen Idempotente

$$
e_{k i}=p_{i} e_{k 1} p_{i}^{-1}
$$

wobei $p_{i}$ zu $\Im_{\langle n+1, \ldots, a\rangle}$ gehört. Damit wird

$$
\begin{aligned}
\psi_{k} & =e_{\langle 1, \ldots, n\rangle}^{-} e_{\langle n+1, \ldots, a\rangle}^{-} \sum_{i=1}^{r} e_{k i} e_{\langle n+1, \ldots, a\rangle}^{-} \psi \\
& =e_{\langle 1, \ldots, n\rangle}^{-} e_{\langle n+1, \ldots, a\rangle}^{-} \sum_{i=1}^{r} p_{i} e_{k 1} p_{i}{ }^{-1} e_{\langle n+1, \ldots, a\rangle}^{-} \psi .
\end{aligned}
$$

Es ist $p_{i}{ }^{-1} e_{\langle n+1, \ldots, a\rangle}^{-}$

$$
= \pm e_{\langle n+1, \ldots, a\rangle}^{-} \pm \text {für } p_{i}^{-1} \quad \begin{aligned}
& \text { gerade } \\
& \text { ungerade }
\end{aligned}
$$

und $e_{\langle n+1, \ldots, a\rangle}^{-} p_{i}=p_{i} e_{\langle n+1, \ldots, a\rangle}^{-}= \pm e_{\langle n+1, \ldots, a\rangle}^{-}$

für $p_{i}$, d. h. auch für $p_{i}{ }^{-1}$ gerade ungerade.

Also $\psi_{k}=r \cdot e_{<1, \ldots, n \searrow}^{-} \quad e_{\langle n+1, \ldots, a\rangle}^{-} e_{k_{1}} \psi$.

Wendet man hierauf irgendwelche Permutationen der $\widetilde{\Xi}_{a}$ an, so kommt man aus dem zu $e_{k i}$ gehörenden Linksideal nicht heraus, d. h. die zugehörige irreduzible Darstellung tritt höchstens einmal auf.

Die folgende Betrachtung über die Dimension der Darstellungsräume wird diese Aussage verschärfen: Ủbt man auf $\psi\left(x_{1}, \ldots, x_{n} \mid x_{n+1}, \ldots, a\right)$ die Operationen $\operatorname{der} \mathfrak{\subseteq}_{a}$ aus, so entstehen $N=\left(\begin{array}{l}a \\ n\end{array}\right)$ linear unabhängige Funktionen, sofern nicht weitere Symmetrieeigenschaften außer der Totalantimetrie unter $\widetilde{\Xi}_{\langle 1, \ldots, n\rangle\langle n+1, \ldots, a\rangle}$ vorhanden sind.

Es sei nun die Annahme gemacht, jede der zugelassenen irreduziblen Darstellungen komme genau einmal vor. Wie groß wird die Dimensionszahl des Darstellungsraumes? Zur Beantwortung dieser Frage sind die Dimensionszahlen der irreduziblen Darstellungen, die zu den zweispaltigen Rahmen, deren erste Spalte $u \geqq n$ Kästchen besitzt, gehören, zu addieren. Ein Rahmen mit der Zeilenlänge $m_{1}, m_{2}, \ldots, m_{r}, m_{1}+m_{2}+\ldots+m_{r}=a$ besitzt

$$
f=a ! \frac{\prod_{i<k}\left(l_{i}-l_{k}\right)}{l_{1} ! l_{2} ! \ldots l_{r} !}
$$

Standardtableaux, wobei

$$
l_{1}=m_{1}+r-1, \quad l_{2}=m_{2}+r-2, \quad l_{r}=m_{r}
$$

ist; $f$ bedeutet gleichzeitig, nach einem oben angeführten Satz, die Dimensionszahl der zum Rahmen gehörenden irreduziblen Darstellungen. Für den Rahmen

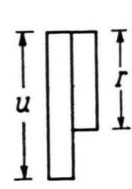

$$
\text { wobei } u=n, n+1, \ldots, a
$$$$
v=a-n, \ldots, 0
$$

sein können,

wird $u=r$ und $f$

mit $\quad l_{1}=2+u-1, \quad l_{2}=2+u-2, \ldots, l_{v}=2+u-v, \quad l_{v+1}=1+u-(v+1), \ldots, l_{u}=1$

$$
\begin{aligned}
f & =a ! \frac{\prod_{k>1}\left(l_{1}-l_{k}\right) \prod_{k>2}\left(l_{2}-l_{k}\right) \ldots \prod_{k>v}\left(l_{v}-l_{k}\right) \prod_{k>v+1}\left(l_{v+1}-l_{k}\right) \ldots\left(l_{u-1}-l_{u}\right)}{l_{2} ! \ldots} l_{v+1} ! \ldots l_{u} ! \\
& =a ! \frac{u !}{v} \cdot \frac{(u-1) !}{v-1} \ldots \frac{(u+1-v) !}{1}(u-v-1) !(u-v-2) ! \ldots 2 ! 1 ! \\
f & =\frac{a !}{v !} \frac{(u+1-v) !}{(u+1) !(u-v) !}=\left(\begin{array}{l}
a \\
u
\end{array}\right) \frac{2 u+1-a}{u+1} .
\end{aligned}
$$


Summiert man über alle möglichen irreduziblen Darstellungen, so ergibt sich

$$
N^{\prime}=\sum_{u=n}^{a}\left(\begin{array}{l}
a \\
u
\end{array}\right) \frac{2 u+1-a}{u+1} .
$$

Behauptung:

$$
N^{\prime}=N=\left(\begin{array}{l}
a \\
u
\end{array}\right) \text {. }
$$

Für $n=a$ ist die Behauptung richtig $N^{\prime}=\left(\begin{array}{l}a \\ a\end{array}\right) \frac{a+1}{a+1}=1$. Angenommen, sie gelte für $n=t$, dann wird für $n=t-1$

$$
\begin{aligned}
N^{\prime} & =\sum_{u=t-1}^{a}\left(\begin{array}{l}
a \\
u
\end{array}\right) \begin{array}{c}
2 u+1-a \\
u+1
\end{array} \\
& =\left[\left(\begin{array}{l}
a \\
u
\end{array}\right) \frac{2 u+1-a}{u+1}\right]_{u=t-1}+\sum_{u=t}^{a}\left(\begin{array}{l}
a \\
u
\end{array}\right) \frac{2 u+1-a}{u-1} \\
& =\left(\begin{array}{c}
a \\
t-1
\end{array}\right) \frac{2 t-1-a}{1}+\left(\begin{array}{l}
a \\
t
\end{array}\right)=\left(\begin{array}{c}
a \\
-1
\end{array}\right),
\end{aligned}
$$

$N^{\prime}=\left(\begin{array}{l}a \\ n\end{array}\right)=N \quad$ gilt also allgemein.

Der Beweis zeigt, daß jede der erlaubten irreduziblen Darstellungen bei der Zerlegung von

$$
\psi\left(x_{1}, \ldots, x_{n} \mid x_{n+1}, \ldots, x_{a}\right)
$$

genau einmal vorkommt, sofern $\psi$ nicht weitere Symmetrieeigenschaften außer der Totalantimetrie unter $\widetilde{\mathfrak{S}}_{\langle 1, \ldots, n\rangle\langle n+1, \ldots, a\rangle}$ besitzt.

b) Die unter $\mathfrak{\Xi}\langle 1, \ldots, n\rangle\langle n+1, \ldots, a\rangle$ total antimetrischen Vektoren

Die Darstellungsräume der erlaubten irreduziblen Darstellungen der $\widetilde{\Xi}_{a}$, von denen man annehmen kann, daß jede bei der Zerlegung von $\psi$ genau einmal vorkommt, werden von Zuständen gebildet, die im allgemeinen unter $\mathbb{S}\langle 1, \ldots, n\rangle\langle n+1, \ldots, a\rangle$ nicht antimetrisch sind. Bei der gewünschten Abbildung interessieren jedoch nur die unter dieser Untergruppe antimetrischen, da diese allein wegen des Pauli-Prinzips realisiert sind.

\section{Es gilt der folgende Satz:}

Jede der erlaubten irreduziblen Darstellungen (die durch Anwendung von $\widetilde{\Xi}_{a}$ auf $\psi$ entstehen) enthält genau einen unter $\widetilde{\Xi}\langle 1, \ldots, n\rangle\langle n+1, \ldots, a\rangle$ antimetrischen $\mathrm{Zu}$ stand.

Der Beweis gründet sich auf ein Reziprozitätstheorem von $F_{\text {robenius, }}$ dem $\mathrm{W}_{\mathrm{EyL}}{ }^{26}$ eine für den vorliegenden Sachverhalt geeignete Form gegeben hat: Betrachtet wird die reguläre Darstellung einer endlichen Gruppe $\mathfrak{G}$. Diese ist natürlich auch eine Darstellung einer Untergruppe $\mathfrak{g}$ von $\mathfrak{G}$. Es wird ausgegangen von einer irreduziblen Darstellung $\gamma_{0}$ von $\mathrm{g}$. Der Darstellungsraum von $\gamma_{0}$ besteht aus gewissen Ringelementen. Wendet man von links die Gruppenoperationen an, so erhält man ein Linksideal; auf diesem wird durch geeignete Zuordnung von Matrizen eine Darstellung $\Gamma$ von $\mathfrak{G}$ induziert. Diese

26 H. WEYL, Gruppentheorie und Quantenmechanik, Verlag S. Hirzel, Stuttgart 1931. kann man in irreduzible Darstellungen $\Gamma_{j}$ zerfällen. Beschränkt man sich auf die Matrizen, welche Elementen von $\mathrm{g}$ zugeordnet sind, so wird jedes einzelne $\Gamma_{j}$ im allgemeinen weiter zerfallen. Kommt dabei $\gamma_{0}$ in $\Gamma_{j}$ vor und wie oft? Hierauf gibt das Reziprozitätstheorem die Antwort: Kommt die irreduzible Darstellung $\Gamma_{j}$ in $\Gamma$ $b_{j}$-mal vor, dann ist auch $\gamma_{0}$ in $\Gamma_{j}$ genau $b_{j}$-mal enthalten. Dieser Satz wird auf das unter der Untergruppe $\widetilde{\Xi}_{\langle 1, \ldots, n\rangle\langle n+1, \ldots, a\rangle}$ totalantimetrische

$$
\psi\left(x_{1}, \ldots, x_{n} \mid x_{n+1}, \ldots, x_{a}\right)
$$

angewandt, für das man zweckmäßig

$$
\psi=e_{\langle 1, \ldots, n\rangle}^{-} e_{\langle n+1, \ldots, a\rangle}^{-} \psi
$$

schreibt. Der zu $\gamma_{0}$ gehörende Darstellungsraum ist hier eindimensional und besteht aus dem Element $\psi$. An Stelle der $\Gamma_{j}$ treten die als erlaubt erkannten irreduziblen Darstellungen von $\gamma_{a}$. Jede kommt nach dem vorangehenden Abschnitt genau einmal vor. Nach dem Reziprozitätstheorem ist daher in jeder genau ein unter $\mathfrak{\Xi}_{: 1, \ldots, n\rangle\langle n+1, \ldots, a\rangle}$ totalantimetrischer Vektor enthalten, w. z. b. w.

\section{c) Die Abbildung}

Aus einer Zusammenfassung der gewonnenen Ergebnisse wird klar, wie die Abbildung aussehen muß. Der Zustandsraum zerfällt in Sektoren $H^{0}, H^{1}, \ldots, H^{a}$ (der untere Index $a$ ist, da Irrtümer ausgeschlossen, weg. gelassen). Liege in $H^{n}$ das Eigenfunktionensystem (i) $\psi^{n}$ vor. Die Zerlegung von ${ }^{(i)} \psi^{n}$ bei festem i nach irreduziblen Darstellungen der Permutationsgruppe

$$
\text { (i) } \psi^{n}=\sum_{k} e_{k}^{(\mathrm{i})} \psi^{n}=\sum_{k}^{(\mathrm{i})} \psi_{k^{n}}
$$

ergibt eine bestimmte Anzahl von irreduziblen Darstellungen, die von $n$ abhängt. Jede dieser Darstellungen kommt nur einmal vor und in jedem dieser Darstellungsräume befindet sich genau ein unter $\Xi_{\langle 1, \ldots, n\rangle\langle n+1, \ldots, a\rangle}$ antimetrischer Zustand.

Man kann sich bei der Abbildung auf die unter $\widetilde{S}_{\langle 1, \ldots, n \backslash\langle n+1, \ldots, a\rangle}$ antimetrischen Zustände beschränken, da nur diese realisiert sind; sie seien in etwas abgeänderter Bedeutung des Index $k$ mit ${ }^{(i)} \psi_{k}^{n}$ bezeichnet.

Die Zuordnung, in der sich das Prinzip der Ladungsunabhängigkeit ausdrückt, sieht so aus :

${ }^{(i)} \psi_{k}^{n} \longleftrightarrow{ }^{(i)} \psi_{k}{ }^{n} \quad$ sofern in $H^{n^{\prime}}$ die $k$-te irreduzible Darstellung vorkommt,

(i) $\psi_{k}{ }^{n} \longleftrightarrow 0 \quad$ sofern in $H^{n^{\prime}}$ die $k$-te irreduzible Darstellung fehlt.

Entsprechend, wenn in $H^{n}$ die $k$-te irreduzible Darstellung fehlt.

So sind die zugeordneten Zustandsvektoren aufgewiesen und damit hat man die Abbildung definiert. Diese Abbildung läßt sich durch Anwendung gewisser Ringelemente von $\widetilde{S}_{a}$ auf $\psi_{k}^{n}$ explizit durchführen. Darauf mag hier verzichtet werden, statt dessen wenden wir uns dem Zusammenhang zwischen der vorliegenden Methode und dem Isotopenspinformalismus zu. 
d) Zusammenhang zwischen der vorliegenden Methode und dem Isotopenspinformalismus

Betrachtet sei ein Beispiel des $a$-Nukleonenproblems, etwa $a=4$.

Die Einteilung des Zustandsraumes in Sektoren und die darin vorkommenden irreduziblen Darstellungen der Permutationsgruppe, durch ihre Rahmen charakterisiert, weisen deutlich auf den Zusammenhang zwischen den irreduziblen Darstellungen der Permutationsgruppe, die in der vorliegenden Methode auftreten, und den irreduziblen Darstellungen der Drehgruppe des Isotopenspins hin. Jede der Zellen enthält ein Multiplett, von oben nach unten Quintett, Triplett, Singulett, so daß die Isotopenspin-Quantenzahlen $t=2,1,0 \mathrm{zu}$ vermuten sind (siehe Abb. 4). Seien zunächst die Isotopenspin-Eigenfunktionen betrachtet. Die Verhältnisse liegen analog den gewöhnlichen Spinfunktionen von mehreren Teilchen ${ }^{27}$. Die beiden Transformationsgruppen

1. Drehungen des Systems im Isotopenspinraum, und

2. Permutationen der Teilchen,

d. h. genauer Permutationen der Eigenschaften der Teilchen, Proton oder Neutron zu sein, sind miteinander vertauschbar, so daß die Darstellungen der Drehgruppe und der Permutationsgruppe sich simultan ausreduzieren lassen. Man kann also solche Eigenvektoren wählen, daß man sie in Rechtecken anzuordnen vermag, z. B. so, daß die Vektoren, die in einer Zeile stehen, sich ineinander nach einer bestimmten irreduziblen Darstellung der Drehgruppe, die für das Rechteck kennzeichnend ist, transformieren, während die Vektoren einer Spalte nach einer bestimmten Darstellung der Permutationsgruppe, die gleichfalls für das Rechteck charakteristisch ist, ineinander übergeführt werden. Dabei treten, wie schon von Überlegungen bei Ortsspinfunktionen her gut bekannt ist, nur irreduzible Darstellungen der Permutationsgruppe, die zu Rahmen mit nicht mehr als zwei Zeilen gehören, auf. Es kommen nur solche zweizeiligen Rahmen vor, bei denen der Zeilenunterschied $g^{\prime} \geqq 2 n-a$ ist. Weiter ist bekannt, daß die Zuordnung von irreduziblen Darstellungen, die sich in dem Auftreten der beschriebenen Rechtecke von Eigenfunktionen ausdrückt, eineindeutig ist. Das heißt, zu jeder irreduziblen Darstellung der Permutationsgruppe gehört genau eine der Drehgruppen, und zwar ist dem Zeilenunterschied $g^{\prime}$ der Spin (hier Isotopenspin) $j=g^{\prime} / 2$ zugeordnet.

Die Zuordnung einer bestimmten irreduziblen Darstellung der Permutationsgruppe (Darstellungen der Permutationsgruppe mit $\Delta$, die zur Drehgruppe mit $D$ bezeichnet) zu einer bestimmten irreduziblen der Dreh- gruppe soll symbolisch so bezeichnet werden:

$$
D_{k} \longleftrightarrow \Delta_{k}^{\prime} \text {. }
$$

Eine weitere Zuordnung von irreduziblen Darstellungen zueinander kommt hinzu: Sei $\psi\left(x_{1}, x_{2}, \ldots, x_{a}\right)$ eine Orts-Spineigenfunktion und $\Omega(1, \ldots, a)$ eine IsotopenSpinfunktion, deren Eigenwert $j_{z}$ dem vorliegenden physikalischen System gemäß gewählt ist, so soll aus $\Psi\left(x_{1}, \ldots, a\right) \Omega(1, \ldots, a)$ nach einer Vorschrift des Isotopenspinformalismus die total-antimetrische Darstellung $\Gamma^{-} \operatorname{der} \Im_{a}$ gebildet werden:

$$
\begin{aligned}
\varphi=c \Sigma & \pm p_{i}\left\{\Psi\left(x_{1}, \ldots, a\right) \Omega(1, \ldots, a)\right\} \\
& + \text { bei geraden Permutationen } \\
& - \text { bei ungeraden Permutationen } \\
& c \text { Normierungsfehler. }
\end{aligned}
$$

Für $\pm p_{i}\{\Psi \cdot \Omega\}$ läßt sich auch ausführlich

$$
\pm p_{i} \Psi\left(x_{1}, \ldots, a\right) p_{i} \Omega(1, \ldots, a)
$$

schreiben. Im Raume der Orts-Spinfunktion $\Psi$ erfahre $\widetilde{S}_{a}$ eine irreduzible Darstellung $\Delta$, im Raume der Isotopenspinfunktion $\Omega$ eine irreduzible Darstellung $\Delta^{\prime}$. Gesucht sind solche $\Delta$ und $\Delta^{\prime}$, da $\beta$ die Produktdarstellung $\Delta \times \Delta^{\prime}$ die Darstellung $\Gamma^{-}$(also $\varphi$ ) enthält. Es müssen $\Delta$ und $\Delta^{\prime}$ assoziiert sein. Einem vorgelegten $\Delta$ ist damit eindeutig ein $\Delta^{\prime}$ zugeordnet, welches die auf die Orts-Spinfunktion ausgeübten Permutationen repräsentiert. Symbolisch:

$$
\Delta \longleftrightarrow \Delta^{\prime} \text {. }
$$

Zusammen mit der ersten Zuordnung erhält man

$$
D \longleftrightarrow \Delta^{\prime} \longleftrightarrow \Delta, \text { d. h. } D \longleftrightarrow \Delta \text {. }
$$

Gehöre $D$ zum Eigenwert $j(j+1)$, so besitzt $\Delta^{\prime}$ einen mit Zeilenunterschied $g^{\prime}=2 j$ und das assoziierte $\Delta$ einen Spaltenunterschied $g=2 j$.

Damit ist gezeigt, wie im Isotopen-Spinformalismus die irreduziblen Darstellungen der Permutationsgruppe im Orts-Spinraum den irreduziblen Darstellungen der Drehgruppe im „Isotopen-Spinraum“ zugeordnet sind.

Beim vorliegenden Verfahren treten dieselben irreduziblen Darstellungen der Permutationsgruppe im OrtsSpinraum auf und die Zuordnung der Isotopen-Spineigenwerte (wie beim Beispiel $a=4$ angegeben) zu den die irreduziblen Darstellungen symbolisch vertretenden Rahmen ist klar.

27 G. Lubwig, Die Grundlagen der Quantenmechanik, Springer-Verlag, Berlin 1954. 